\title{
BMJ Global Health Effect of a home-visiting parenting program to promote early childhood development and prevent violence: a cluster-randomized trial in Rwanda
}

\author{
Sarah KG Jensen, ${ }^{1}$ Matias Placencio-Castro, ${ }^{2}$ Shauna M Murray, ${ }^{1}$ \\ Robert T Brennan, ${ }^{1,3}$ Simo Goshev, ${ }^{4}$ Jordan Farrar, ${ }^{1}$ Aisha Yousafzai, ${ }^{5}$ \\ Laura B Rawlings, ${ }^{6}$ Briana Wilson, ${ }^{6}$ Emmanuel Habyarimana, ${ }^{7}$ Vincent Sezibera, ${ }^{8}$ \\ Theresa S Betancourt ${ }^{1}$
}

To cite: Jensen SKG, Placencio-Castro M, Murray SM, et al. Effect of a home-visiting parenting program to promote early childhood development and prevent violence: a cluster-randomized trial in Rwanda. BMJ Global Health 2021;6:e03508. doi:10.1136/ bmjgh-2020-003508

Handling editor Valery Ridde

- Additional material is published online only. To view please visit the journal online (http://dx.doi.org/10.1136/ bmjgh-2020-003508)

Received 21 July 2020 Revised 3 November 2020 Accepted 23 December 2020

Check for updates

(C) Author(s) (or their employer(s)) 2021. Re-use permitted under CC BY-NC. No commercial re-use. See rights and permissions. Published by BMJ.

For numbered affiliations see end of article.

Correspondence to Dr Theresa S Betancourt; Theresa.betancourt@bc.edu

\section{ABSTRACT}

Introduction Families living in extreme poverty require interventions to support early-childhood development (ECD) due to broad risks. This longitudinal cluster randomised trial examines the effectiveness of Sugira Muryango (SM), a home-visiting intervention linked to Rwanda's social protection system to promote ECD and reduce violence compared with usual care (UC).

Methods Families with children aged 6-36 months were recruited in 284 geographical clusters across three districts. Cluster-level randomisation (allocated 1:1 SM:UC) was used to prevent diffusion. SM was hypothesised to improve child development, reduce violence and increase father engagement. Developmental outcomes were assessed using the Ages and Stages Questionnaire (ASQ3) and the Malawi Development Assessment Tool (MDAT) and anthropometric assessments of growth. Violence was assessed using questions from UNICEF Multiple Indicators Cluster Survey (MICS) and Rwanda Demographic and Health Surveys (DHS). Father engagement was assessed using the Home Observation for Measurement of the Environment. Blinded enumerators conducted interviews and developmental assessments.

Results A total of 541 SM families and 508 UC families were enrolled and included in the analyses. Study attrition ( $2.0 \%$ children; $9.6 \%$ caregivers) was addressed by hot deck imputation. Children in SM families improved more on gross motor $(\mathrm{d}=0.162,95 \% \mathrm{Cl} 0.065$ to 0.260$)$, communication ( $\mathrm{d}=0.081,95 \% \mathrm{Cl} 0.005$ to 0.156 ), problem solving ( $\mathrm{d}=0.101,95 \% \mathrm{Cl} 0.002$ to 0.179$)$ and personal-social development $(\mathrm{d}=0.096,95 \% \mathrm{Cl}-0.015$ to 0.177 ) on the ASQ-3. SM families showed increased father engagement $(\mathrm{OR}=1.592,95 \% \mathrm{Cl} 1.069$ to 2.368), decreased harsh discipline (incidence rate ratio, IRR $=0.741,95 \% \mathrm{Cl} 0.657$ to 0.835 ) and intimate partner violence (IRR $=0.616,95 \% \mathrm{Cl}: 0.458$ to 0.828$)$. There were no intervention-related improvements on MDAT or child growth.

Conclusion Social protection programmes provide a means to deliver ECD intervention.

Trial registration number NCT02510313.

\section{Key questions}

What is already known?

- Early child development interventions can improve developmental outcomes of children in severe poverty.

- Common risk exposures affecting child development in low-resource settings include malnutrition, parental stress and poor mental health, violence and poor hygiene.

What are the new findings?

- Sugira Muryango is a home-visiting-based parenting intervention that addresses known risks to child development in a low-resource setting.

- We reach the poorest families in Rwanda by layering a parenting intervention into an on-going government-organised social protection programme.

What do the new findings imply?

- This trial demonstrates that a scalable home-visiting intervention delivered by lay workers to recipients of a poverty-targeted social protection programme can promote early-childhood development (ECD) and prevent family violence when conducted within a robust system of supervision.

- We add to the evidence for the efficacy and effectiveness of lay worker delivered community-based approaches to promotion of ECD using a strong, well-powered design.

\section{INTRODUCTION}

Families living in extreme poverty require attention to risks to healthy child development, such as low stimulation, poor hygiene and nutrition, violence and neglect. Violence is a major factor affecting healthy development, but family violence is rarely addressed in early-childhood development (ECD)-focused interventions. ${ }^{1}$ Moreover, the typical focus of ECD interventions is on 
mothers, ${ }^{2}$ limiting the ability to work with the full home environment, including male caregivers. Despite an increasing call to move beyond focusing on mothers in initiatives to promote caregivers' capabilities to provide enriched environments for children, ${ }^{3}$ few intervention studies have focused on integrating promotion of father engagement ${ }^{2}$ and family violence prevention ${ }^{4}$ into programmes that focus on common ECD elements such as nutrition, early learning and nurturing care. ECD interventions are broadly defined as programmatic efforts aimed at supporting healthy child development across domains of growth, developmental milestones, cognition, language and socioemotional development. Violence prevention may include education on harmful effects of direct and observed violence on children, and introduction of nonviolent strategies for resolving parental disputes and disciplining children. Previous home-visiting interventions conducted in low and middle-income countries demonstrate the value of integrated approaches that link parenting interventions to other ECD-promoting programmes including economic strengthening programmes such as cash transfer programmes, ${ }^{56}$ nutritional interventions, ${ }^{7-9}$ primary healthcare ${ }^{10}$ and maternal mental health interventions. ${ }^{11}$ However, evidence regarding interventions that combine a focus on improving ECD outcomes and reducing violence against children is sparse or of low quality. A 2018 systematic review identified six studies up to $2014 .^{12}$ Only two of these studies involved randomisation, and both the randomised trial $(n=45)$ and the cluster randomised trial (CRT) ( $\mathrm{n}$ of clusters $=5$ ) lacked sufficient numbers for randomisation to assure balance across treatment and control conditions. We located an additional five studies from January 2015 to May 2020 that featured both ECD and child violence outcomes. ${ }^{13-17}$ Among these recent studies, the rigour of the study design varied considerably. Two trials were randomised, but had insufficient enrolment to assume balance through randomisation alone, and no stratification or matching was employed. ${ }^{13}{ }^{14}$ More specifically, one small randomised controlled trial of the Triple P-positive parenting intervention in Indonesia observed significant improvements in parenting practices, parental stress and child behavioural problems but did not examine developmental milestones. ${ }^{13}$ A randomised trial from Liberia found that a parenting intervention successfully reduced harsh discipline and improved caregiver-child interactions, but did not observe any improvements in child health or development. ${ }^{14}$ Two studies used a cluster randomised design, each with 24 clusters. One of these was a home-visiting programme enrolling pregnant women in South Africa, and used matching at the cluster level. This study found that the intervention had a significant effect on child health, growth and language, but did not find significant intervention effects on violence in the home. ${ }^{15}$ The other CRT, which was set in Jamaica, was a preschool-based programme that did not use matching and found positive intervention effects on teacher and child behaviours, but did not examine outcomes related to children's developmental milestones. ${ }^{16}$ Finally, a quasi-experimental study using matching of counties across two provinces in China found positive effects of a parenting intervention that integrated components from the nurturing care framework with violence prevention on suspected developmental delay, and found that these effects were partially accounted for by improvements in positive discipline, cognitive stimulation and child growth. ${ }^{17}$ Looking at the previous literature, evidence from even the more rigorous trails has been somewhat mixed. For example, two studies including the CRT from South Africa had significant findings in only one area (ie, ECD or violence reduction)..${ }^{14} 15$

Programmes targeting ECD and violence reduction have yet to be evaluated through rigorous, large-scale studies employing randomised designs to add to the evidence of impact on child outcomes. Government social protection programmes present a potentially innovative platform for integrating home-visiting interventions to promote ECD interventions, improving nurturing care, health and nutrition, and reducing violence, thus providing a vehicle for targeting poor and vulnerable populations, and combining income support with a focus on behaviours and investments in human capital. ${ }^{5}$ The Rwandan government established a social protection programme called the 'Vision 2020 Umurenge Programme' in 2007 to address poverty and human-capital related disparities by offering direct support to poor families with young children and public works-based support. ${ }^{18}$ Moreover, in 2017, the government established the National Early Childhood Development Programme to coordinate and expand an array of ECD initiatives, including ECD centres and home-visiting interventions for the most vulnerable families. In this paper, we evaluate the effectiveness of the Sugira Muryango (SM) (Strengthen the Family) intervention, a relatively brief (12 sessions delivered weekly over 3 months) comprehensive home-visiting intervention for families with young children to promote ECD and reduce family violence as implemented by communitybased coaches and delivered in combination with Rwanda's social protection programme compared with those receiving usual care (UC) and social protection. We have previously reported intervention effects on caregiver behaviours immediately postintervention. ${ }^{19}$ This paper reports on results from a 12-month follow-up. In line with our theory of change (figure 1), we hypothesise that participation in SM will be associated with improved child development outcomes 1 year after the intervention ended because previously reported changes in caregiver behaviours have succeed in building a healthy, stimulating and safe environment that supports children's achievement of developmental milestones. Primary outcomes examined at 12-month follow-up are therefore children's developmental milestones, father engagement in childcare and violence reduction (harsh discipline, maternal victimisation to intimate partner violence and paternal perpetration of violence). Given the emphasis 


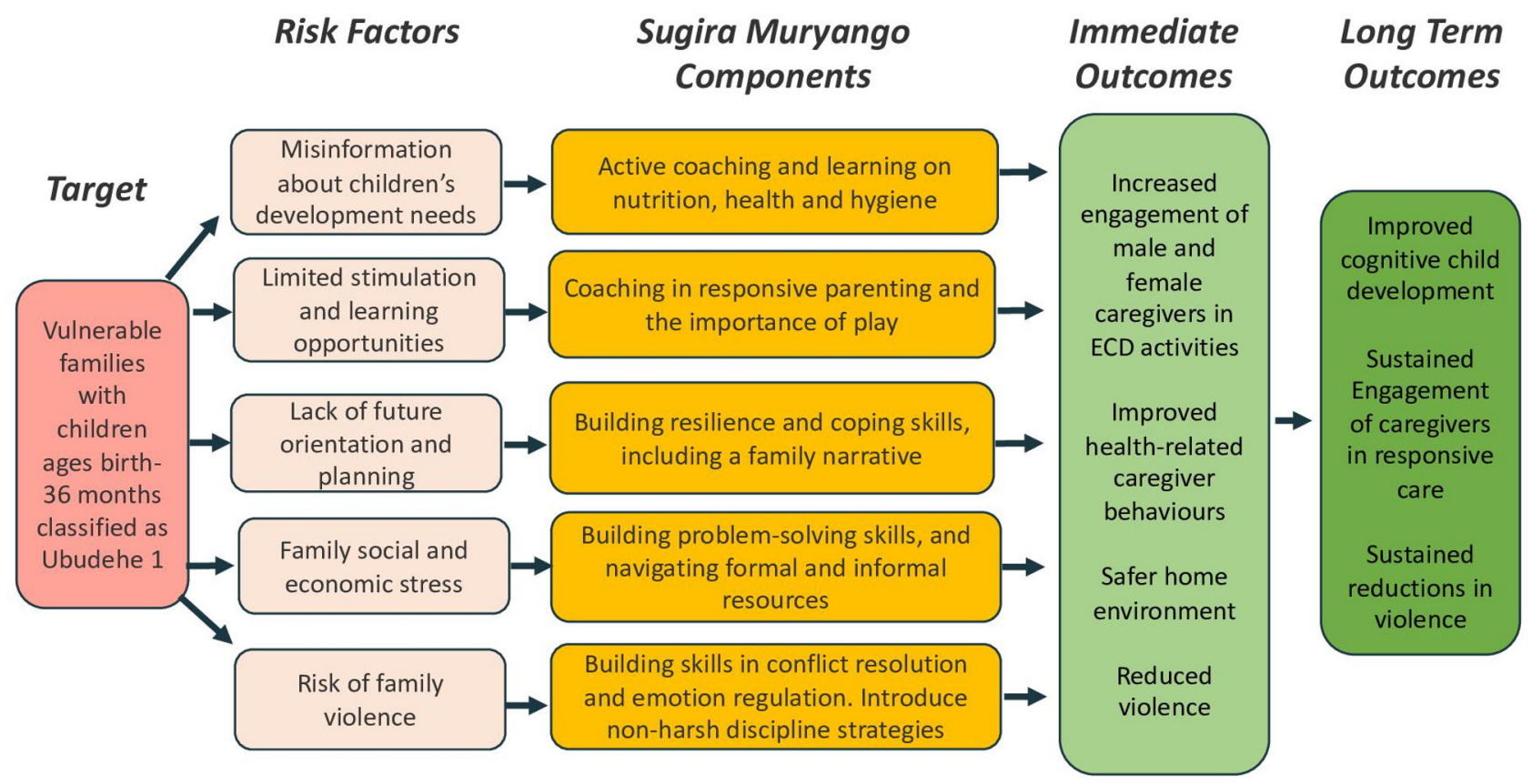

Figure 1 Sugira Muryango theory of change. Reprinted with remission from Betancourt et al ${ }^{24}$-https://www.ncbi.nlm.nih.gov/ pmc/articles/PMC7201751/.

on father engagement and violence reduction, we also hypothesise that such effects will be sustained 1 year after the intervention ended. Measures related to child growth were included as primary outcomes in the clinical trial registration, but later redefined as secondary outcomes in the study protocol in recognition that even programmes that focus on and provide nutritional supplementation struggle to achieve improvements on child growth.

\section{METHODS \\ Study design}

We used a stratified CRT design to test SM's effect on children's motor, cognitive, language and social development, and physical growth, as well as family-level violence and father engagement in care among families enrolled in the social protection programme. The use of a CRT over simple randomised assignment of families was to reduce the threat of diffusion of elements of SM within the setting of Rwandan villages. The CRT was conducted within three Rwandan districts selected based on the presence of the social protection programme and paying attention to other on-going interventions in order to minimise overlap with other large-scale ECD interventions that could bias results. The study protocol can be found with the clinical trials registration cited above. Both coaches and enumerators were blind to the family's intervention status at the time of baseline assessments. Enumerators were also not informed about the family's assignment status during the two post-intervention assessments.

\section{Participants}

Families were eligible for inclusion in the study if they: (1) were eligible for either the classic public works, which provided cash for intensive manual labour, or the newer expanded public works, which provided cash for more flexible, less strenuous work and thus all belonged to the most extreme level of poverty in the government's household-ranking system, Ubudehe 1; (2) had one or more child(ren) aged 6-36 months and (3) were willing to engage in a home-visiting intervention. The age range of 6-36 months was defined to supplement existing ECD service for children in Rwanda, which are most widely available for children aged 5 years and older. Exclusion criteria for caregivers were a severe crisis such as psychosis or suicide attempts or severe cognitive impairment which would prevent the caregiver from responding to the interviews. All adult caregivers gave written informed consent for themselves and their eligible children. The primary caregiver was defined as the caregiver who self-identified as knowing the child best, most often the mother.

\section{Randomisation and masking}

Families were enrolled between 9 February 2018 and 27 April 2018 (see figure 2 for timeline). Government staff in Nyanza, Ngoma and Rubavu districts provided lists of households that were eligible for the government's public works programme. Geographically defined clusters were created as non-overlapping and comprising at least 30 families identified as eligible for the classic public works programme or at least ten families identified as eligible for the newer expanded public works programme, with some clusters containing both $\geq 30$ classic public works and $\geq 10$ expanded public works-eligible households. Clusters were formed from one or more contiguous villages so that one coach could provide services to all eligible families in that cluster maintaining a caseload of five families per coach. Within the same cluster, villages 
Household Identification and Enrollment (January -April 2018)

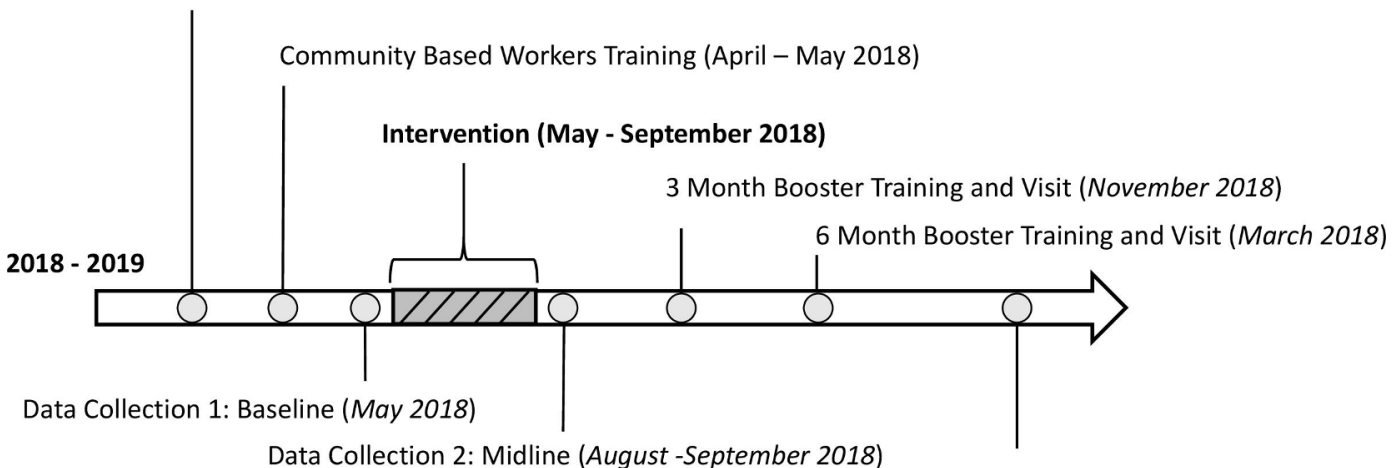

Data Collection 2: Midline (August -September 2018)

Data Collection 3: Follow-up (August -September 2019)

Figure 2 Study timeline.

were selected to be as proximate as possible and as distal from other clusters as possible, such that after random assignment to SM or UC, risk of diffusion to UC families would be minimised. Given the limited reach of expanded public works programming at the time of the CRT, $100 \%$ of clusters containing at least 10 expanded public works families were sampled for participation in the study. Clusters containing classic public works families (including combined clusters) were randomly sampled for inclusion in the CRT until the target sample size of $\geq 1040$ households. Randomisation was completed at the cluster level within strata defined by public works type (expanded public works only, combined expanded public works/classic public works and classic public works only) and geographical sector. By strata, clusters were assigned random numbers and placed on a ranked list. On the randomly ranked list, the first half of clusters were assigned to SM. In case of an uneven number of clusters per strata, randomisation was used to round the number assigned to SM up or down. After cluster assignment, households were invited to participate in the study. We retained clusters if at least five families in the classic public works strata or at least one family in the expanded public works strata enrolled and had at least one child aged 6-36 months. The final sample encompassed 198 clusters: 48 expanded PW-only clusters, 38 expanded $\mathrm{PW} /$ classic PW clusters, and 112 classic PW-only clusters were retained (figure 3). Given the ongoing roll-out of

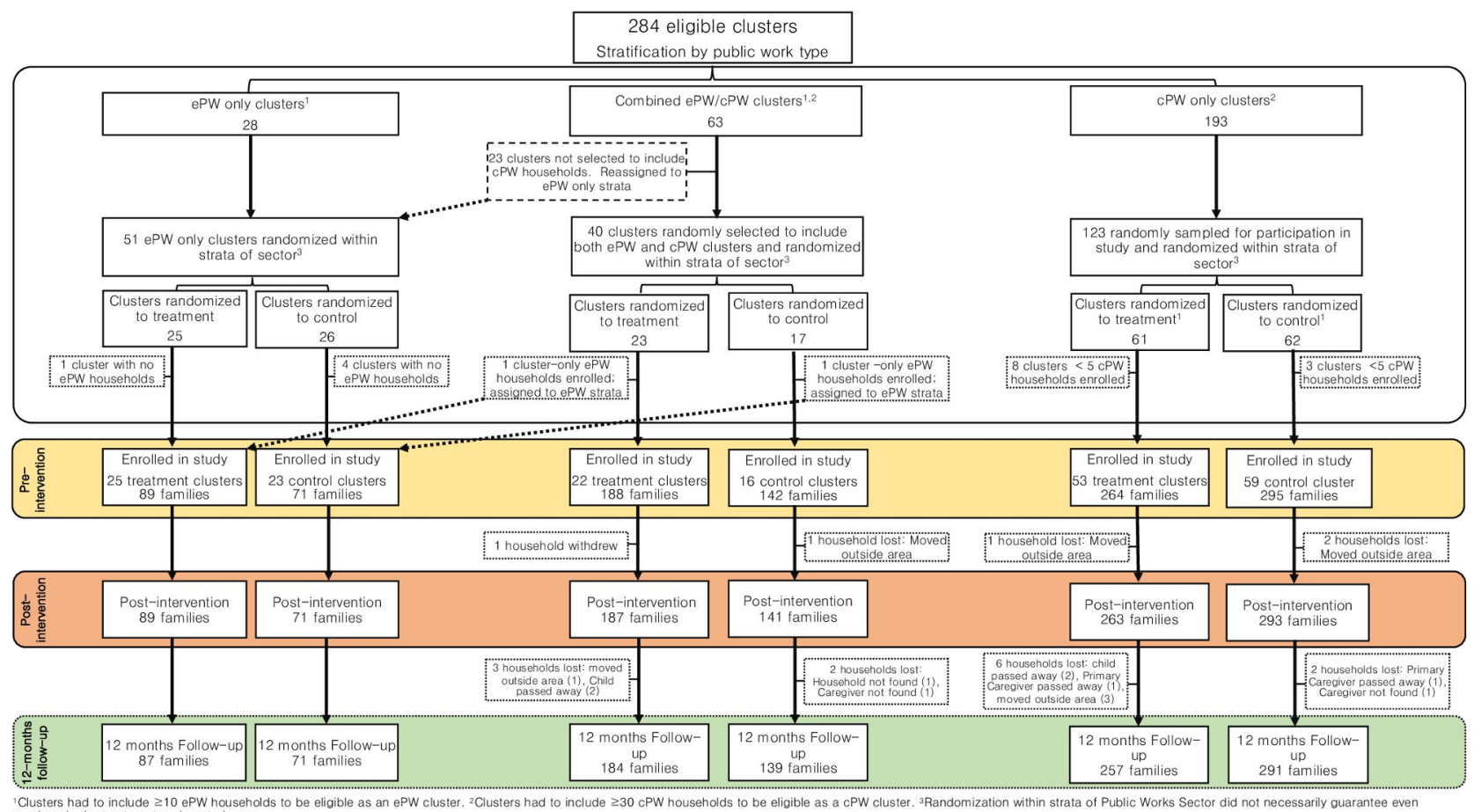

Figure 3 Cluster sampling strategy and flow chart of participants in the Sugira Muryango trial. Although each cluster had a $50 \%$ chance of being assigned to receive Sugira Muryango, we were not guaranteed an equal number of Sugira Muryango and usual care clusters because randomisation occurred within relatively small strata that sometimes contained an odd number of clusters. ePw, expanded public works; cPW, classic public works. 
the expanded public works programme during the design phase for the CRT, it was not possible to have expanded public works families make up half of our sample, thus, calculations were based on an assumption of 91 expanded public works clusters and 104 classic public works clusters with five households per cluster assigned to SM and UC conditions. After the calculation, further adjustments were made, by adding combined clusters of expanded public works clusters and classic public works randomly allocated to condition. Adding more classic public works clusters allowed us to maintain power to test SM versus UC on the primary hypotheses. The CRT enrolled a total of 1049 households. Randomisation by cluster allocated $\mathrm{n}=508$ families to $\mathrm{UC}$ and $\mathrm{n}=541$ families to SM. The overall average number of households per cluster was 6.2 (SD: 2.4), with an average number of households per cluster of 6.0 (SD: 2.2) for UC families and 6.3 (SD: 2.6) for SM families.

\section{Intervention component}

The intervention was implemented from 7 May 2018 to 14 September 2018. The intervention was staggered by district to ensure it started within 2 weeks of baseline data collection. SM is a home-visiting-based intervention that uses psychoeducation and active coaching of caregivers to promote responsive caregiving, nutrition, hygiene and nonviolent interactions among household members (see figure 1 and online supplemental table 1 for overview of intervention content). Active participation and engagement of both female and male caregivers (as present in the home) in childcare and household-related decision making was encouraged throughout the programme. Each visit included a 15-min active play session, in which caregivers received live feedback on parent-child interactions to support and enhance responsive care following the UNICEF and the WHO Care for Child Development package $^{20}$ and Nurturing Care Framework. ${ }^{21}$ Flexible scheduling and messaging during the visits encouraged engagement of male caregivers in play and nurturing care along with female caregivers. The coaches also helped families navigate formal resources such as government programmes to promote child health and nutrition including supplemental nutrition for malnourished children and informal supports such as those from neighbours and extended family to address issues such as family conflict and housing insecurity. The core evidence-based parenting curriculum, which makes up the backbone of the SM intervention, was originally developed and tested in HIV/AIDS-affected Rwandan families with schoolaged children, which included nonviolent parenting and caregiver conflict resolution strategies. ${ }^{22}$ During previous pilot studies, ${ }^{19}{ }^{23}$ a non-HIV/AIDS-specific version of the SM home-visiting programme with an ECD focus was developed by integrating UNICEF/WHO Care for Child Development ${ }^{20}$ materials with additional framing consistent with the WHO and UNICEF Nurturing Care Framework. $^{21} \mathrm{SM}$ comprises 12 modules (see online supplemental table 1 for details) that are delivered by coaches at a pace of about one module per week. Each module takes an average of $60 \mathrm{~min}$.

Home-visiting modules facilitated by the coaches involved participation of female and male caregivers (as present) in interaction with their child(ren) whereby 'serve and return' (ie, responsive) interactions were pointed out and coached to encourage responsive and stimulating parent-child interactions. Other caregivers and children in the household were welcome to participate. Sessions were facilitated in participating families' homes, unless contraindicated due to privacy concerns. Three-month and 6-month booster sessions occurred from 18 November 2018 to 7 December 2018 and 4 March to 30 March 2019, respectively. The aim of each booster visit was to reconnect with families, identify and address ongoing challenges, and engage caregivers in an 'active play' session as modelled in each home-visiting session (described below); each booster visit was approximately 1 hour.

The coaches delivering SM were selected from the local community using a three-step process: (1) nomination from community members; (2) a phone screening and (3) an intensive in-person interview in which applicants were given a curriculum vignette of a challenging family situation and asked to explain the vignette, instructed to explain an image shown from the curriculum, which depicted family unity, nutrition, father engagement and early stimulation, and administered a writing test to capture demographic information and answer brief questions to identify any prior experience they may have had delivering community-based programmes (see online supplemental table 2 for detail on training, supervision and incentive practices). Prior to the start of the intervention, coaches participated in a 3-week training ( 120 hours), followed by a multilayered approach to supervision that included in-person supervision during the first 3 weeks of programme delivery, weekly telephone supervision (approximately 12 hours total) and monthly in-person group supervision. Additionally, weekly in-person peer support groups, facilitated by lead coaches, were held to complement supervision strategies and promote transfer of audiorecordings for fidelity monitoring using lead coaches' computers (approximately 15 hours total). Coaches received training on confidentiality and risk of harm protocols. Coaches received a monthly stipend of RWF28 000 (caseload of five households).

Families in both the intervention households and the UC condition were eligible for the social protection public works programme and services as usual from the Rwandan government and its partners. There was no incentive associated with participation in SM but all families (SM and UC) received a stipend (RWF5000 equal to $3 \mathrm{~kg}$ of rice) after each data collection time point. Data collection was carried out by trained local enumerators working for an independent research firm, who were blind to the intervention status of the families. Enumerators signed confidentiality agreements embedded in their contracts and were trained to identify cases of risk 
of harm. Assessments were conducted in Kinyarwanda in the family's home, except anthropometric measurements which were taken at the local health centre. All questionnaires were developed from pilot intervention research and were forward-translated and back-translated from English to Kinyarwanda following standard WHO procedures. ${ }^{23}$ Data were entered on Android tablets. Baseline assessments were conducted between 23 April 2018 and 1 June 2018, with immediate postintervention assessments conducted between 13 August 2018 and 30 September 2018, and 12-month follow-up assessments conducted between 19 August 2019 and 30 September 2019.

\section{Outcome measures}

The primary caregiver provided information about the household, including family composition and assets. Per our conceptual model (figure 1), the post-treatment (3 month) outcomes focused on change in caregiver practices, including parent-child interactions, diet, health/ hygiene, family functioning and family violence. ${ }^{24}$ The 12-month primary outcomes, assessed here, focused on changes in children's motor, language, cognitive and social development, as well as violence reduction, and father's engagement in caregiving. Secondary outcomes were child stunting (ie, child height for age equal to or more than 2 SD below age and sex norms) and related anthropometric assessments.

Child motor, language, cognitive and social development was assessed using the Ages and Stages Questionnaire (ASQ-3), ${ }^{25}$ and the Malawi Developmental Assessment Tool (MDAT). ${ }^{26}$ The ASQ-3 is a series of age-specific questionnaires designed to screen for developmental delay of children in the areas of gross motor skills, fine motor skills, communication and problem solving. We administered the ASQ-3 through interviews with the primary caregiver. No developmental cut-offs for the ASQ-3 have been validated for use in Rwanda, thus, we used continuous scoring using standard guidelines for obtaining norm-referenced z-scores using means and SD from reference samples in South Africa and Zambia. ${ }^{27}$ The MDAT is an observational, task-based measure used to asses child development across domains of gross motor, fine motor, language and socioemotional development. The MDAT was developed for use in Malawi with the aim to be culturally appropriate for use in rural Africa. ${ }^{26}$ The MDAT assessment was performed by trained enumerators. We obtained norm-referenced z-scores using means and SD from the Sanitation Hygiene Infant Nutrition Efficacy study from Zimbabwe. ${ }^{28}$

Children's anthropometric growth was assessed using measures of standardised height-for-age (HAZ), standardised weight-for-age (WAZ), standardised weight-forheight (WHZ) and middle upper arm circumference (MUAC). In coordination with community health workers and local leaders, enumerators conducted child growth assessments in centralised locations using locally sourced MUAC tapes and height boards and scales purchased in the USA. The scales were calibrated every day before data collection using a weighing bag as a standard weight, to check that the scales read the same weight at the beginning of every day. If the measurements differed in any way, the team checked the equipment and changed batteries if needed to make sure the scales were accurate. Height was measured in centimetres to the nearest $0.1 \mathrm{~cm}$. Weight was measured in kilograms to the nearest $1 \mathrm{~g}$. Middle upper arm circumference was measured in centimetres to the nearest $0.1 \mathrm{~cm}$. Standardisation was done using WHO Anthro Survey Analyser software. ${ }^{29}$

Father engagement was measured using an item from the Home Observation for Measurement of the Environment Inventory, ${ }^{30}$ namely 'father spends time every day caring for the child' with response options 'yes/no' reported by the primary caregiver. Violent and nonviolent discipline practices were assessed using the UNICEF Multiple Indicator Cluster Survey Child Development and Child Disciplinary modules, as reported by the primary caregivers. ${ }^{31}$ Exposure to violent disciplinary practices included being shouted or screamed at, called demeaning names, shaken, spanked, slapped or beaten. These exposures were summed to create a continuous score. Intimate partner violence was assessed by the Rwanda Demographic and Health Survey's Domestic Violence Module among caregivers who reported being currently married, cohabitating or in a relationship. ${ }^{32} \mathrm{We}$ report experiences of physical or sexual abuse victimisation among female caregivers and perpetration of abuse among male caregivers within the last 3 months. Adverse events were defined as severe malnutrition or illness of the child, caregiver suicidality, reports of severe violence or the death of a caregiver or child.

\section{Sample size calculation}

Data from two previous pilot studies were used to estimate power and determine sample size for a 0.18 minimum detectable standardised effect size (d) on child development and violence reduction outcomes for the postintervention and 12-month follow-up period assuming power of 0.8 and a standard two-tailed alpha level of $\mathrm{p}<0.05$ using Optimal Design V.3.01. Estimated intraclass correlation for geographical clusters was 0.03 for parent-child interactions based on published pilot data, ${ }^{193}$ and a baseline survey conducted in collaboration with UNICEF. ${ }^{33}$

\section{Statistical analysis}

Effectiveness of SM was determined based on significant differences in the slope of the response variable for SM families compared with UC. Data were analysed by fitting a linear mixed effect model for continuous outcomes where the primary predictors were treatment group (SM vs UC), time as a continuous variable, and their two-way interaction all of which were included as fixed effects. The model had three levels of nesting: children/ caregivers are measured within measurement waves, and measurement waves are nested within randomisation cluster or region. Since we expect region-level and time-level effects, subject-specific slopes and intercepts 
Table 1 Estimated difference-in-difference coefficients for continuous, binary and count outcomes

\begin{tabular}{|c|c|c|c|c|}
\hline \multirow[b]{2}{*}{ Outcome } & \multicolumn{2}{|c|}{$\begin{array}{l}\text { Growth models } \\
\text { (Average change over time in } \\
\text { Sugira Muryango compared } \\
\text { with usual care) }\end{array}$} & \multirow{2}{*}{$\begin{array}{l}\begin{array}{l}\text { Baseline to } \\
\text { postintervention } \\
\text { difference† }\end{array} \\
\text { Marginal effect }(95 \% \mathrm{Cl})\end{array}$} & \multirow{2}{*}{$\begin{array}{l}\text { Baseline to 12-month } \\
\text { follow-up difference } \\
\text { Marginal effect }(95 \% \mathrm{Cl})\end{array}$} \\
\hline & $\begin{array}{l}\text { Difference-in- } \\
\text { difference }^{\star}(95 \% \mathrm{Cl})\end{array}$ & $\begin{array}{l}P \\
\text { value }\end{array}$ & & \\
\hline \multicolumn{5}{|l|}{ Child development outcomes } \\
\hline ASQ-3 Gross motor z-score & $0.294(0.118$ to 0.470$)$ & 0.001 & $0.298(0.150$ to 0.446$)$ & $0.597(0.300$ to 0.893$)$ \\
\hline ASQ-3 Fine Motor z- score & $0.004(-0.119$ to 0.127$)$ & 0.95 & $0.061(-0.043$ to 0.165$)$ & $0.122(-0.086$ to 0.330$)$ \\
\hline ASQ-3 Communication z- score & 0.139 (0.009 to 0.268$)$ & 0.034 & $0.129(0.020$ to 0.238$)$ & $0.259(0.041$ to 0.476$)$ \\
\hline ASQ-3 Prob. Solving z- score & 0.159 (0.035 to 0.282$)$ & 0.012 & 0.189 (0.085 to 0.293$)$ & 0.379 (0.171 to 0.587$)$ \\
\hline ASQ-3 Social Emotional z- score & 0.148 (0.023 to 0.273$)$ & 0.020 & $0.122(0.017$ to 0.228$)$ & 0.245 (0.0342 to 0.455$)$ \\
\hline MDAT Gross motor z- score & $-0.003(-0.101$ to 0.095$)$ & 0.951 & $0.028(-0.055$ to 0.111$)$ & $0.055(-0.111$ to 0.221$)$ \\
\hline MDAT fine motor $z$ - score & $0.070(-0.058$ to 0.198$)$ & 0.283 & $0.097(-0.012$ to 0.205$)$ & $0.193(-0.023$ to 0.410$)$ \\
\hline MDAT language z- score & $-0.022(-0.126$ to 0.080$)$ & 0.668 & $-0.016(-0.103$ to 0.072$)$ & $-0.032(-0.206$ to 0.143$)$ \\
\hline MDAT socioemotional z- score & $0.073(-0.016$ to 0.164$)$ & 0.108 & $0.060(-0.016$ to 0.136$)$ & $0.121(-0.031$ to 0.272$)$ \\
\hline \multicolumn{5}{|l|}{ Father engagement } \\
\hline Father engagement in childcareł & $1.591(1.069,2.368)$ & 0.022 & $1.565(1.091,2.244)$ & $2.449(1.191,5.037)$ \\
\hline \multicolumn{5}{|l|}{ Child growth outcomes } \\
\hline Height-for-age & $-0.019(-0.067$ to 0.029$)$ & 0.44 & $-0.033(-0.074$ to 0.007$)$ & $-0.067(-0.148$ to 0.0144$)$ \\
\hline Weight-for-age & $-0.031(-0.071$ to 0.009$)$ & 0.139 & $-0.038(-0.072$ to -0.003$)$ & $-0.075(-0.143$ to -0.007$)$ \\
\hline Weight-for-height & $-0.016(-0.069$ to 0.037$)$ & 0.551 & $-0.015(-0.059$ to 0.029$)$ & $-0.030(-0.119$ to 0.0592$)$ \\
\hline Middle upper arm circumference & $-0.057(-0.113$ to -0.001$)$ & 0.046 & $-0.036(-0.083$ to 0.011$)$ & $-0.072(-0.167$ to 0.0227$)$ \\
\hline \multicolumn{5}{|l|}{ Violence and safety } \\
\hline Harsh discipline $\S$ & 0.741 (0.657 to 0.835$)$ & $<0.001$ & 0.774 (0.688 to 0.870$)$ & 0.632 (0.510 to 0.783$)$ \\
\hline $\begin{array}{l}\text { Victimisation (female } \\
\text { caregivers)§, ๆ }\end{array}$ & 0.616 (0.458 to 0.828$)$ & 0.001 & $0.616(0.425$ to 0.893$)$ & $0.442(0.238$ to 0.820$)$ \\
\hline Perpetration (male caregivers) $§, ~ * *$ & $0.604(0.325$ to 0.110$)$ & 0.110 & 0.897 (0.708 to 1.134$)$ & 0.842 (0.559 to 1.266$)$ \\
\hline
\end{tabular}

*Assesses the significance of the 'difference-in-difference' or 'time-by-treatment' interaction coefficient.

†Difference in marginal means estimates between Sugira Muryango and usual care at each time point.

$\ddagger$ Among households with a father $(\mathrm{N}=524)$, Displayed as OR.

§From MICS: UNICEF's Multiple Indicator Cluster Survey, displayed as incidence rate ratio.

IAmong female caregivers reporting a current intimate partner at baseline $(n=523)$, displayed as incidence rate ratio.

${ }^{\star \star}$ Among male caregivers reporting a current intimate partner at baseline $(n=450)$, displayed as incidence rate ratio.

ASQ-3, Ages and Stages Questionnaire; MDAT, Malawi Development Assessment Tool.

were modelled as random effects nested within randomisation clusters. Additionally, the type of public works programme the family participated in (a stratifier) was modelled as a fixed effect. Binary outcomes were estimated using generalised linear mixed models with a binomial distribution and logistic link, and count outcomes were analysed using multilevel mixed-effects negative binomial models. Other details of the model are the same as the linear model described above. We defined significance as observing $p<0.05$ (two tailed) for the interaction term between treatment and time. We also report adjusted effect sizes as the marginal effects from the mixed effects models (marginal effect for continuous outcomes and ORs for binary outcomes) for the baseline to immediate postintervention and baseline to 12-month follow-up assessments (table 1). Analyses were conducted in StataMP V.16. ${ }^{34}$ Under intention-totreat assumptions, we replaced lost cases using multiple imputation via the HOTDECK plug-in module for Stata ${ }^{35-38}$ by randomly selecting five cases matched on sex, public works programme type, household structure, age and treatment group for child outcomes; caregiver cases were matched on public works programme type, age, educational achievement and treatment group. This is preferred to carrying the last observation forward, a method now widely understood to be problematic. ${ }^{39}$ Quality assurance checks were conducted during data collection using proprietary audit algorithms to review survey metadata and flag unusual submissions for further investigation by a trained data manager and senior field supervisor. A random sample of $10 \%$ of all surveys were automatically recorded and reviewed by a supervisor to 
ensure enumerators followed appropriate data collection protocols.

\section{Baseline equivalence and modification of intervention effects by child sex}

Per Sex and Gender Equity in Research guidelines, we explored baseline equivalence and potential differential intervention effects by child sex by rerunning all models including a main effect of gender, a treatmentby-gender interaction, a time point-by-gender interaction and a treatment-by-gender-by-time point interaction.

\section{RESULTS}

Baseline data were collected on 1084 children and 1498 caregivers and intimate partners. Loss to-follow-up at 12 months (2.0\% for children; $9.6 \%$ for caregivers) and item-level missing data $(0.1 \%-2.5 \%)$ were low across the outcomes. Table 2 provides sample descriptive statistics. Caregivers ranged in age from 18 to 84 years at baseline and were most often the biological mother $(n=950)$, the biological father $(n=433)$, or a grandparent $(n=96)$. A total of $70 \%(\mathrm{n}=1048)$ of the included caregivers were primary caregivers and $30 \%$ were secondary caregivers. Among the primary caregivers, $98.9 \%$ were female. Among the secondary caregivers $98.4 \%$ were male. At baseline, $64 \%$ of the caregivers were married or cohabitating, $61 \%$ of the families reported high levels of food insecurity and $48 \%$ of the children were stunted as defined by a standardised HAZ score below -2 SD of the reference population at baseline.

Results from the linear growth models and marginal effects are shown in table 1. Raw means and SD are reported in the online supplemental table 3; intra-class correlations for main outcomes are displayed in online supplemental table 4 .

We observed differences in change over time between SM and UC on the ASQ-3. Intervention families improved more across the domains of gross motor $(\beta=0.294,95 \%$ CI: 0.118 to 0.470$)$, communication $(\beta=0.13995 \%$ CI 0.009 to 0.268$)$, problem solving $(\beta=0.159,95 \%$ CI 0.035 to 0.282$)$ and personal-social development ( $\beta=0.148,95 \%$ CI 0.023 to 0.273 ). We did not see any significant group differences in change on fine motor development.

We did not observe any significant group differences in changes on the MDAT across any of the domain scores: gross motor, fine motor, language and socioemotional development.

Regarding child growth, at 12-month follow-up, we did not observe any significant intervention effects on HAZ, WAZ, WHZ or MUAC (all 95\% CIs crossed zero).

Among families with a father in the household, father engagement increased more in families receiving SM compared with UC (OR=1.592, 95\% CI 1.069 to 2.368). Families receiving SM also showed a greater decrease in the use of harsh discipline (IRR $=0.741,95 \% \mathrm{CI}$
0.657 to 0.835$)$. Among those in dual-caregiver households, we found that female caregivers receiving SM showed a greater decrease in intimate partner violence (IRR $=0.616,95 \%$ CI 0.458 to 0.828 ). However, there was no significant reduction of male caregiver reported perpetration of violence towards their partner.

Adverse events were reported to SM staff by coaches and enumerators within 24 hours of identification. From baseline to the 12-month follow-up assessment, 28 risk of harm cases were reported among intervention families while 27 occurred in the UC group (details in online supplemental file 1). These households were retained in the analyses under intention to treat.

\section{Effects of child sex}

Analyses examining baseline equivalence and intervention effects by child sex did not reveal any differences in intervention-related improvements in male versus female children.

\section{DISCUSSION}

The primary hypotheses of greater improvements in child development, violence reduction and father engagement among families receiving the intervention compared with $\mathrm{UC}$ was supported for violence reduction, father engagement and developmental outcomes measured by the parent-reported ASQ-3. For family violence (ie, maternal reports of intimate partner violence and harsh discipline) and father engagement the hypothesis of greater improvement was supported at both points of intervention follow-up. We did not see significantly greater improvements on the MDAT, however, and there was no evidence of greater improvement in the secondary anthropometric growth outcomes. We have previously shown immediate post-treatment (3 months) effects of SM on responsive caregiving, caregiver engagement in play activities with the child, decreased use of violent discipline and improvements in the mental health of male and female caregivers. ${ }^{24}$ We also observed immediate pretreatment to post-treatment increases in caregiver behaviours to support healthy child development including increased dietary diversity, hygiene behaviours and healthcare seeking for sick children among participants in SM relative to families in UC. ${ }^{24}$ Our theory of change (figure 1) would predict that such changes in parenting behaviours, in turn, would be lead to improvements in long-term ECD and growth outcomes. The combination of significant caregiver behavioural change immediately post-treatment, and improvement in developmental milestones and sustainment of violence reduction at 12-month postintervention follow-up supports this theoretical foundation of SM. By linking an ECD programme into an existing social protection infrastructure, SM was able to identify and reach the most vulnerable population of caregivers in Rwanda, those classified as being most vulnerable according to the government's poverty ranking system. The linkage with 
Table 2 Characteristics of study participants at enrolment. Continuous variables reported as means (SD)

\begin{tabular}{|c|c|c|c|c|}
\hline & \multicolumn{2}{|c|}{ Classic public works (cPW) } & \multicolumn{2}{|c|}{ Expanded public works (ePW) } \\
\hline & $\begin{array}{l}\text { Sugira } \\
\text { Muryango+cPW }\end{array}$ & cPW only & $\begin{array}{l}\text { Sugira Muryango } \\
+\mathrm{ePW}\end{array}$ & ePW only \\
\hline Households $(n=1049)$ & $n=374$ & $\mathrm{n}=374$ & $\mathrm{n}=167$ & $n=134$ \\
\hline High food insecurity & 239 (63.9\%) & $229(61.2 \%)$ & $104(62.3 \%)$ & 70 (52.2\%) \\
\hline Children $(n=1084)$ & $n=386$ & $\mathrm{n}=384$ & $n=173$ & $n=141$ \\
\hline Average age in months & $21.0(8.14)$ & $21.8(8.6)$ & $20.8(8.2)$ & $22.3(8.4)$ \\
\hline \multicolumn{5}{|l|}{ Health status and well-being } \\
\hline Stunted (standardised height-for-age<2) & $184(47.7 \%)$ & 178 (46.4\%) & $85(49.1 \%)$ & $75(53.2 \%)$ \\
\hline Wasted (standardised weight-for-height $<2$ ) & $13(3.4 \%)$ & $9(2.3 \%)$ & $8(4.6 \%)$ & $2(1.4 \%)$ \\
\hline Underweight (standardised weight-for-age<2) & $63(16.3 \%)$ & $71(18.5 \%)$ & $30(17.3 \%)$ & $27(19.1 \%)$ \\
\hline Screens positive, disability or developmental delay & $110(28.6 \%)$ & $111(29.0 \%)$ & $57(32.9 \%)$ & $38(27.1 \%)$ \\
\hline \multicolumn{5}{|l|}{ Disciplinary practices } \\
\hline Any violent punishment & $184(47.7 \%)$ & $180(47.0 \%)$ & $83(48.0 \%)$ & $59(41.8 \%)$ \\
\hline Caregivers $(n=1498)$ & $\mathrm{n}=555$ & $\mathrm{n}=564$ & $\mathrm{n}=211$ & $\mathrm{n}=168$ \\
\hline Primary caregiver & $374(67.4 \%)$ & $374(66.3 \%)$ & $166(78.7 \%)$ & $134(79.8 \%)$ \\
\hline Female & $371(66.9 \%)$ & $372(65.9 \%)$ & $166(78.7 \%)$ & $134(79.8 \%)$ \\
\hline Average age in years & $34.5(9.7)$ & $35.7(10.3)$ & $36.3(10.6)$ & $37.5(12.7)$ \\
\hline (Range) & $(18-79)$ & $(19-75)$ & $(18-79)$ & $(18-84)$ \\
\hline \multicolumn{5}{|l|}{ Marital Status } \\
\hline Single, separated, divorced, widowed & $171(30.8 \%)$ & $166(29.4 \%)$ & $117(55.5 \%)$ & $91(54.2 \%)$ \\
\hline \multicolumn{5}{|l|}{ Relationship with child } \\
\hline Biological mother & $341(61.4 \%)$ & $338(59.9 \%)$ & $152(72.0 \%)$ & $119(70.8 \%)$ \\
\hline Biological father & $179(32.3 \%)$ & $183(32.4 \%)$ & $44(20.9 \%)$ & $27(16.1 \%)$ \\
\hline Adoptive mother & $2(0.4 \%)$ & $1(0.2 \%)$ & $0(0.0 \%)$ & $0(0.0 \%)$ \\
\hline Stepfather & $1(0.2 \%)$ & $4(0.7 \%)$ & $0(0.0 \%)$ & $5(3.0 \%)$ \\
\hline Stepmother & $1(0.2 \%)$ & $0(0.0 \%)$ & $0(0.0 \%)$ & $0(0.0 \%)$ \\
\hline Aunt/uncle & $3(0.5 \%)$ & $2(0.4 \%)$ & $0(0.0 \%)$ & $0(0.0 \%)$ \\
\hline Grandparents & $28(5.0 \%)$ & $36(6.4 \%)$ & $15(7.1 \%)$ & $17(10.1 \%)$ \\
\hline \multicolumn{5}{|l|}{ Educational Attainment } \\
\hline No school/don't know & $112(20.2 \%)$ & $132(23.4 \%)$ & $60(28.4 \%)$ & $38(22.6 \%)$ \\
\hline$<6$ years & $275(49.5 \%)$ & $252(44.7 \%)$ & $97(46.0 \%)$ & $88(52.4 \%)$ \\
\hline$\geq 6$ years primary & $88(15.9 \%)$ & $89(15.8 \%)$ & $26(12.3 \%)$ & $21(12.5 \%)$ \\
\hline Secondary/vocational school & $80(14.4 \%)$ & $91(16.1 \%)$ & $28(13.3 \%)$ & $21(12.5 \%)$ \\
\hline \multicolumn{5}{|l|}{ Health and safety } \\
\hline Maternal victimisation violence, last 3 months ${ }^{*}$ & $78(39.8 \%)$ & $73(35.3 \%)$ & $15(29.4 \%)$ & $15(36.6 \%)$ \\
\hline Paternal perpetration violence, last 3 months ${ }^{*}$ & $38(21.2 \%)$ & $41(22.3 \%)$ & $10(23.3 \%)$ & $4(12.5 \%)$ \\
\hline
\end{tabular}

Binary variables reported as frequency (\%).

*Among mothers $(n=495)$ and fathers $(n=438)$ who are married or cohabitating.

a social protection programme may introduce several other benefits. For example, integration of a parenting intervention within social protection systems may result in higher participation rates and stronger social buy-in around the parenting programme. Moreover, combined ECD and social protection programmes may benefit from several synergistic effects because social protection programmes tend to provide important benefits such as access to health insurance and healthcare, nutritional counselling and/or supplements, and cash for work that can be directed towards food, and other vital househod expenses, all of which impact a family's ability to provide a safe and healthy environment for a child.

The use of active coaching allowed SM to involve all family members, including fathers and other male caregivers present in the household in nurturing care as reflected in increased father engagement. Indeed, we note that father engagement is one of the strongest 
effects of SM, especially postintervention where fathers in SM families were 2.5 times more likely to have engaged with their child in the past 24 hours, compared with control fathers. Flexible scheduling and messaging about the importance of fathers in ensuring a nurturing and safe environment for young children to grow and thrive resulted in both high module attendance by fathers and a significant relative increase in fathers' involvement in childcare. This adds to the important and growing literature on ways to engage fathers in ECD interventions and childcare responsibilities. Previous work from Uganda found that use of father-only sessions, exploitation of men's pre-existing motivation to improve their children's behaviour, and interactive delivery helped to engage fathers ${ }^{40}$

SM also maintained effects on reduced violence, including reduced violent discipline and reduced maternal reports of intimate partner violence observed immediately postintervention and again 1year after the intervention ended. ${ }^{24}$ We did not retain the reduction of paternal-reported perpetration of intimate partner violence observed immediately postintervention. Violence reduction is a key aim of SM and is achieved through a combination of psychoeducation and coaching on topics ranging from mindfulness meditation techniques, conflict resolution, alternative strategies to harsh discipline, the harmful effect of harsh discipline, the benefits of responsive care and father engagement in childcare. We believe that the use of active coaching and instruction was central in establishing persistent changes in caregiver behaviours. The inclusion of violence reduction was motivated by previous identification of violence as a key challenge to ECD among young children in Rwanda. ${ }^{33}$ During the intervention, coaches became aware of several incidents of violence in the families they served and worked closely with their supervisor and village officials to assess and resolve such situations and to link families with existing resources.

Key strengths of the study compared with prior related studies include the rigorous, well-powered CRT design and randomisation, including the blinding of the enumerators to treatment condition and geographical separation of SM locations from UC. The use of a stratified randomised clustered design with larger numbers of both clusters and families is a marked improvement over studies recently reviewed systematically ${ }^{12}$ and found in our own review of more recent studies. ${ }^{13-17}$

With regard to cognitive developmental outcomes, the results from the ASQ-3 and MDAT were not consistent. The MDAT was new to the Rwandan setting; it is possible that revision of items and other refinements may be necessary to ensure its sensitivity to change over time in that environment. Although both the ASQ-3 (caregiver report) and MDAT (direct assessment) were originally developed as screening tools to detect developmental delay, not growth trends, the ASQ-3 has been widely used in previous intervention studies in settings as diverse as Rwanda, ${ }^{23}$ China ${ }^{17}$ and Peru ${ }^{41}$ and found to be sensitive to effects of home-visiting and parenting interventions. ${ }^{42-44}$ The observed effects on the parent-reported ASQ-3, in the absence of similar effects on observational measures, may suggest that parenting interventions change or sensitise parents' perception of their child's behaviours and abilities, and thus affect the parents' ability to provide parent reports over time. Moreover, although intervention and control families were exposed to the same test batteries, parents receiving the intervention may feel more encouraged to report on their children's developmental milestones because they know they received an intervention. Interestingly, a similar discrepancy between parent report vs an observer rating was found in an evaluation of Peru's home-visiting Cuna Mas programme wherein a programme delivery fidelity measure was found to correlate significantly with the ASQ-3 scores, but not with the Bayley scales ${ }^{45}$ a gold-standard observational measure administered by selected highly trained psychology graduates. ${ }^{41}$

SM also did not lead to improvements in child growth. While the $95 \%$ CI crosses zero for all estimated effects on growth outcomes, we saw a small negative trend towards lower MUAC in the SM children at the 12-month follow-up, but this was not significant. Several factors may explain the absence of positive intervention effects on children's anthropometric outcomes. At baseline, $48 \%$ of the children were stunted, which is known to decrease the opportunity to change a child's growth trajectory, because the effects of early malnutrition are difficult to reverse. ${ }^{46}$ Although the curriculum addresses child feeding, nutrition and hygiene with the aim of improving children's health and growth, a behavioural intervention with active coaching on nurturing care may not be enough to cause measurable improvements in anthropometric growth, particularly among extremely vulnerable households and already malnourished children. For example, parental training in diet among parents in extreme poverty without additional direct nutrition support may not be adequate to overcome past and present malnutrition. In future research, the impact of SM on child growth may be intensified if combined with other programmes, such as child-focused conditional cash transfers, agricultural and food supplementation, health, water, sanitisation and hygiene programmes. Future research should also test the proposed mechanistic pathways of change in child outcomes via changes in caregiver behaviours put forward in our theory of change. Moreover, future analyses looking at potential moderators of intervention outcomes (beyond child sex) should be explored to understand whether certain subpopulations benefit more or less from the intervention.

Our results are consistent with other studies that have examined child development and parental behaviour outcomes in the context of combined ECD and government social protection programmes. In Colombia, an ECD programme that delivered psychosocial stimulation (and micronutrient supplementation) to beneficiaries of the Familias en Acción conditional cash transfer 
programme yielded promising short-term impacts on parenting behaviours and child cognitive development. ${ }^{5}$ Specifically, the parenting intervention, which consisted of weekly home visits by beneficiary-elected female community leaders over an 18-month period, was associated with statistically significant improvements in cognition (effect size $0.26 \mathrm{SD}$ ) and receptive language (effect size $0.22 \mathrm{SD}$ ). Similarly, results from a randomised controlled trial of a home-based parenting intervention delivered by local NGOs to households participating in Niger's unconditional cash transfer programme (Niger Safety Nets) also lend support for integrated approaches to supporting the holistic development of children living in poverty. Among families who participated in the ECD intervention, a combination of monthly home visits and small group meetings, they found that children had moderate improvements in socioemotional development, and parents interacted with their children around activities such as storytelling, counting or drawing more frequently and were less likely to use harsh disciplinary practices. ${ }^{46}$ Overall, these along with current findings suggest that integrated ECD and social protection programmes hold promise for improvements in child cognitive development and parenting behaviours. Evidence for anthropometric gains in combined ECD and social protection programmes, however, remains elusive. The potential for combined ECD and social protection such as cash transfer programmes to have synergistic effects remains inconclusive and understudied since most studies use social protection as a platform for identifying their beneficiaries and don't include an ECD-only arm. SM, a brief, comprehensive ECD intervention delivered by lay coaches, successfully targeted and reached families living in extreme poverty and provided an opportunity for parenting support to complement income support through two public works programmes. SM is designed to be deployment-focused, relatively brief and delivered by lay workers with strong fidelity monitoring and quality improvement-focused supervision. Scaling out the programme to reach large numbers of families in extreme poverty might best be achieved through the utilisation of a pre-existing government workforce. In Rwanda, such a work force includes the inshuti z'umuryango (friends of the family) child protection worker and other community health workforces. Linkage of ECD programmes to the existing social protection platform, as explored in this study, has several advantages; it targets the most disadvantaged, supports equity, and coordinates with other interventions (eg, food supplementation, conditional cash transfers). Combined provision of social protection and ECD intervention may also have several synergistic effects because even greater gains, especially on anthropometric growth may be possible if the programme were integrated explicitly with a programme providing nutritional supplementation. Such synergistic effects will be important to unpack in future research. From an implementation standpoint, coordination across sectors (health and social protection) need to be explored further. Supervision in these systems would need to be addressed to support the workforce to deliver and sustain quality in delivery of the programme which likely requires a different set of competencies. Testing implementation strategies for scaling out the intervention and innovations in structures for sustainment including attention to quality improvement models to support sustained high-quality supervision will be critical future research. A number of study limitations should be noted. First, given the large sample size and focus on vulnerable families, some families enrolled in this study may also have been eligible for, and participated in, other interventions beyond SM and the public works programme. During the selection of target districts, we spoke with a broad range of implementors and funders of related programmes to map ongoing interventions and we selected the targeted districts based on minimal overlap with other interventions. Still, we cannot rule out the possibility that some families may have participated in other interventions that could influence results, although this should not differ between SM and control families. We also note that this study tested the linkages of an ECD intervention with a social protection programme. Synergistic effects of ECD intervention and social protection may mean that findings from the current study do not necessarily generalise to poor families who do not have access to social protection programmes. A second limitation is that key outcomes related to violence in the home and child development assessed on the ASQ is based on parental report. This always introduces some potential for reporting bias. In particular, participation in the study and intervention may have sensitised parents to observing changes in these outcomes.

\section{CONCLUSION}

This CRT integrated SM into Rwanda's social protection programme as a platform for targeting poor and vulnerable families with the potential for transitioning the SM programme to scale. In particular, the programme had a large and persistent effect on engaging fathers in childcare. Moreover, we also saw smaller but significant effects on violence reduction including intimate partner violence and harsh discipline as well as child development. With continued attention to quality and fidelity, evidence-based interventions such as SM can be scaled out across settings, providing the government of Rwanda and others with a critical tool for helping to break intergenerational cycles of poverty and violence.

\footnotetext{
Author affiliations

${ }^{1}$ School of Social Work, Boston College, Chestnut Hill, Massachusetts, USA ${ }^{2}$ Lynch School of Education and Human Development, Boston College, Chestnut Hill, Massachusetts, USA

${ }^{3}$ Women's Study Research Center, Brandeis University, Waltham, Massachusetts, USA

${ }^{4}$ Academic Research Services, Boston College, Chestnut Hill, Massachusetts, USA ${ }^{5}$ Department of Global Health and Population, Harvard T.H. Chan School of Public Health, Boston, Massachusetts, USA

${ }^{6}$ The World Bank, Washington, District of Columbia, USA
} 
${ }^{7}$ FXB Rwanda, Kigali, Rwanda

${ }^{8}$ Center for Mental Health, University of Rwanda, Kigali, Rwanda

Acknowledgements The work was made possible by the collaboration of the University of Rwanda Center for Mental Health, the Ministry of Gender and Family Promotion, the National Child Development Agency (formerly the National Early Childhood Development Program and the National Commission for Children), the Local Administrative Entities Development Agency, and the advisory committee comprised of representatives from the Ministry of Local Government, National Commission for Children, Rwanda Biomedical Centre, Ministry for Gender and Family Promotion, National Early Childhood Development Program and the University of Rwanda School of Nursing.Early Childhood Development Program

Contributors TSB conceptualised the study, obtained funding, led intervention development, provided supervision and review of the manuscript. SKGJ had full access to the data and lead the analyses, interpreted results and lead the writing of the manuscript. MPC conducted data analyses, created tables, and contributed to data interpretation and writing and had full access to the data. SMM oversaw intervention implementation, data collection and made significant intellectual contributions to the manuscript content. RTB contributed to study concept and design, statistical consultation and drafting of the manuscript. SG provided expert advice and help with data imputation and analysis. JF oversaw the integrated fidelity monitoring and supervision process and provided critical review of manuscript content.AY contributed subject matter expertise and critical review of manuscript content. LBR and BW contributed to policy dialog management with government counterparts, study design, securing funding, data interpretation and writing. EH oversaw intervention implementation and policy dialog management with local government. VS contributed to intervention adaptation, facilitated contact and dialog with local partners and made significant intellectual contributions to the intervention and manuscript content.

Funding World Bank Early Learning Partnership, Strategic Impact Evaluation Fund, World Bank Japan Trust Fund, USAID Rwanda, Network of European Foundations and ELMA Foundation.

Disclaimer The World Bank Group (WBG) authors contributed to study design and writing of the manuscript. The findings, interpretations, and conclusions expressed in this paper are entirely those of the authors. They do not necessarily reflect the views of the WBG, its Board of Executive Directors, or the governments they represent. The WBG does not guarantee the accuracy of the information included in this work. The remaining funders, USAID Rwanda, ELMA Foundation, and the Network of European Foundations played no role in study design, data collection, data analysis, data interpretation, or writing of the report.

Competing interests None declared.

Patient and public involvement statement Design of the intervention was carried out with input and routine consultation from stakeholders at the Ministry of Gender and Family Promotion and the National Early Childhood Development Program in Rwanda. We engaged with village leaders and the Ministry of Local Government (MINALOC), which runs the Poverty Reduction Strategy, the Vision Umurenge Program, prior to and during the study implementation to discuss recruitment of participants and to inform them of the process and outcomes. Representatives from the data collection firm, Laterite, and our collaborators at the University of Rwanda, were closely involved in developing and refining the research-questions, study design, selection of measures and assessment tools, and ethical procedures. During implementation, we worked closely with our fieldbased implementation partner, FXB-Rwanda, who provided training and supervision to the community-based coaches to discuss ongoing implementation and emerging issues. At the end of the study, we communicated results at a local dissemination event which included all key stakeholders, including the Government of Rwanda and other organisations that implement child development programs in Rwanda. "The event included a facilitated discussion with program stakeholders, including local government officials and study interventionists, as well as a presentation, led by the Principal Investigator, on program outcomes, lessons learned and next steps.

Patient consent for publication Not required.

Ethics approval Procedures and research protocols related trial (registration number NCT02510313) received ethics approval from the Harvard T. H. Chan School of Public Health (IRB16-1570) and Boston College Institutional Review Boards (19.017), as well as the Rwanda National Ethics Committee (no approval number provided), National Commission for Science and Technology (no approval number provided), and the National Institute of Statistics of Rwanda (no approval number provided).

Provenance and peer review Not commissioned; externally peer reviewed.
Data availability statement Data are available on request. Data can be made available on request and will be made publicly available via the World Bank's data catalog at a later date.

Supplemental material This content has been supplied by the author(s). It has not been vetted by BMJ Publishing Group Limited (BMJ) and may not have been peer-reviewed. Any opinions or recommendations discussed are solely those of the author(s) and are not endorsed by BMJ. BMJ disclaims all liability and responsibility arising from any reliance placed on the content. Where the content includes any translated material, BMJ does not warrant the accuracy and reliability of the translations (including but not limited to local regulations, clinical guidelines, terminology, drug names and drug dosages), and is not responsible for any error and/or omissions arising from translation and adaptation or otherwise.

Open access This is an open access article distributed in accordance with the Creative Commons Attribution Non Commercial (CC BY-NC 4.0) license, which permits others to distribute, remix, adapt, build upon this work non-commercially, and license their derivative works on different terms, provided the original work is properly cited, appropriate credit is given, any changes made indicated, and the use is non-commercial. See: http://creativecommons.org/licenses/by-nc/4.0/.

\section{REFERENCES}

1 Mikton C, MacMillan H, Dua T, et al. Integration of prevention of violence against children and early child development. Lancet Glob Health 2014;2:e442-3.

2 Jeong J, Obradović J, Rasheed M, et al. Maternal and paternal stimulation: Mediators of parenting intervention effects on preschoolers' development. J Appl Dev Psychol 2019;60:105-18.

3 Knaul FM, Bustreo F, Horton R. Countering the pandemic of genderbased violence and maltreatment of young people: the Lancet Commission. Lancet 2020;395:98-9.

4 Ward CL, Wessels IM, Lachman JM, et al. Parenting for lifelong health for young children: a randomized controlled trial of a parenting program in South Africa to prevent harsh parenting and child conduct problems. J Child Psychol Psychiatry 2020;61:503-12.

5 Attanasio OP, Fernández C, Fitzsimons EOA, et al. Using the infrastructure of a conditional cash transfer program to deliver a scalable integrated early child development program in Colombia: cluster randomized controlled trial. BMJ 2014;349:g5785.

6 Lachman J, Wamoyi J, Spreckelsen T, et al. Combining parenting and economic strengthening programmes to reduce violence against children: a cluster randomised controlled trial with predominantly male caregivers in rural Tanzania. BMJ Glob Health 2020;5:e002349.

7 Walker SP, Chang SM, Vera-Hernández M, et al. Early childhood stimulation benefits adult competence and reduces violent behavior. Pediatrics 2011;127:849-57.

8 Yousafzai AK, Obradović J, Rasheed MA, et al. Effects of responsive stimulation and nutrition interventions on children's development and growth at age 4 years in a disadvantaged population in Pakistan: a longitudinal follow-up of a cluster-randomised factorial effectiveness trial. Lancet Glob Health 2016;4:e548-58.

9 Hamadani JD, Huda SN, Khatun F, et al. Psychosocial stimulation improves the development of undernourished children in rural Bangladesh. J Nutr 2006;136:2645-52.

10 Powell C, Baker-Henningham H, Walker S, et al. Feasibility of integrating early stimulation into primary care for undernourished Jamaican children: cluster randomised controlled trial. BMJ 2004;329:89.

11 Singla DR, Kumbakumba E, Aboud FE. Effects of a parenting intervention to address maternal psychological wellbeing and child development and growth in rural Uganda: a community-based, cluster-randomised trial. Lancet Glob Health 2015;3:e458-69.

12 Efevbera Y, McCoy DC, Wuermli AJ, et al. Integrating early child development and violence prevention programs: a systematic review. New Dir Child Adolesc Dev 2018;2018:27-54.

13 Sumargi A, Sofronoff K, Morawska A. A Randomized-Controlled trial of the triple P-Positive parenting program seminar series with Indonesian parents. Child Psychiatry Hum Dev 2015;46:749-61.

14 Puffer ES, Green EP, Chase RM, et al. Parents make the difference: a randomized-controlled trial of a parenting intervention in Liberia. Glob Ment Health 2015;2:e15.

15 Tomlinson M, Rotheram-Borus MJ, le Roux IM, et al. Thirty-sixmonth outcomes of a generalist Paraprofessional perinatal home visiting intervention in South Africa on maternal health and child health and development. Prev Sci 2016;17:937-48.

16 Baker-Henningham $\mathrm{H}$, Walker $\mathrm{S}$. Effect of transporting an evidencebased, violence prevention intervention to Jamaican preschools on 
teacher and class-wide child behaviour: a cluster randomised trial. Glob Ment Health 2018;5:e7.

17 Zhou S, Zhao C, Huang X, et al. The effect of a community-based, integrated and nurturing care intervention on early childhood development in rural China. Public Health 2019;167:125-35.

18 Republic of Rwanda Local Administrative Entities Development Agency (LODA). Environmental and social management framework for the social protection investment project financing operation, 2018. Available: https://www.undp.org/content/undp/en/home/ search.html?q=2020+Umurenge+Program+\%28VUP\%29\%0A

19 Betancourt TS, Franchett E, Kirk CM, et al. Integrating social protection and early childhood development: open trial of a family home-visiting intervention, Sugira Muryango. Early Child Dev Care 2020;190:219-35.

20 World Health Organization, UNICEF. Care for child development: improving the care for young children, 2012. Available: https:// www.who.int/maternal_child_adolescent/documents/care_child_ development/en/

21 UNICEF, WHO. The world bank. nurturing care for early child development: a framework for helping children survive and thrive to transform health and human potential. Geneva, 2018. Available: https://apps.who.int/iris/bitstream/handle/10665/272603/ 9789241514064-eng.pdf

22 Betancourt TS, Ng LC, Kirk CM, et al. Family-Based promotion of mental health in children affected by HIV: a pilot randomized controlled trial. J Child Psychol Psychiatry 2017;58:922-30.

23 Barnhart DA, Farrar J, Murray SM, et al. Lay-worker delivered home visiting promotes early childhood development and reduces violence in Rwanda: a randomized pilot. J Child Fam Stud 2020;29:1804-17.

24 Betancourt TS, Jensen SKG, Barnhart DA, et al. Promoting parentchild relationships and preventing violence via home-visiting: a pre-post cluster randomised trial among Rwandan families linked to social protection programmes. BMC Public Health 2020;20:621.

25 Squires J, Bricker D. Ages \& stages questionnaires, third edition (ASQ-3): a parent-completed child-monitoring system, 2009. Available: https://brookespublishing.com/product/asq-3/

26 Gladstone M, Lancaster GA, Umar E, et al. The Malawi developmental assessment tool (MDAT): the creation, validation, and reliability of a tool to assess child development in rural African settings. PLoS Med 2010;7:e1000273.

27 Hsiao C, Richter L, Makusha T, et al. Use of the ages and stages questionnaire adapted for South Africa and Zambia. Child Care Health Dev 2017;43:59-66.

28 Gladstone MJ, Chandna J, Kandawasvika G, et al. Independent and combined effects of improved water, sanitation, and hygiene (wash) and improved complementary feeding on early neurodevelopment among children born to HIV-negative mothers in rural Zimbabwe: substudy of a cluster-randomized trial. PLoS Med 2019;16:e1002766.

29 World Health Organization. WHO Anthro survey analyser, 2020. Available: https://www.who.int/childgrowth/software/en/
30 Caldwell BM, Bradley RH. Home observation for measurement of the environment: administration manual. Tempe, AZ: Family \& Human Dynamics Research Institute, Arizona State University, 2003.

31 UNICEF. MICS5 questionnaire for children under five, 2013. Available: http://mics.unicef.org/tools?round=mics5

32 National Institute of Statistics of Rwanda, ORC Macro. Rwanda demographic and health survey 2005. Kigali, Rwanda, 2006. Available: https://dhsprogram.com/pubs/pdf/FR183/FR183.pdf

33 UNICEF, Imbuto Foundation. Early childhood development and family services: baseline evaluation in 20 sites in Rwanda, 2015. Available: https://www.unicef.org/evaldatabase/files/ECD_and_F Baseline_Evaluation Rwanda.pdf 34 Statacorp. StataMP 16. 2019

34 Statacorp, StataMP 16. Release 16. College Station, TX: StataCorp LLC, 2019.

35 Reilly M. Data analysis using hot Deck multiple imputation. The Statistician 1993;42:307-13.

36 Little RJA. Missing-Data adjustments in large surveys. J Bus Econ Stat 1988;6:287-96.

37 Lachin JM. Fallacies of last observation carried forward analyses. Clin Trials 2016;13:161-8.

38 Mander A, Clayton D. HOTDECK: Stata module to impute missing values using the hotdeck, 1999. Available: https://ideas.repec.org/c/ boc/bocode/s366901.htm

39 Siu GE, Wight D, Seeley J, et al. Men's Involvement in a Parenting Programme to Reduce Child Maltreatment and Gender-Based Violence: Formative Evaluation in Uganda. Eur J Dev Res 2017;29:1017-37.

40 Araujo MC, Dormal M, Rubio-Codina M. Quality of parenting programs and child development outcomes: the case of Peru's Cuna Mas. Washington D.C. Peru: Ministerio de Educación del Perú, 2018.

41 Atukunda P, Muhoozi GKM, van den Broek TJ, et al. Child development, growth and microbiota: follow-up of a randomized education trial in Uganda. J Glob Health 2019;9:010431.

42 Worku BN, Abessa TG, Wondafrash M, et al. Effects of homebased play-assisted stimulation on developmental performances of children living in extreme poverty: a randomized single-blind controlled trial. BMC Pediatr 2018;18:29.

43 Mohammed H, El-Gibaly O, Monazea E, et al. The effect of a maternal training programme on early childhood development in Egypt. East Mediterr Health J 2019;25:575-82.

44 Bayley N. Bayley scales of infant and toddler development. (3rd ED. San Antonio, TX: The Psychological Corporation, 2006.

45 Rubio-Codina M, Attanasio O, Meghir C, et al. The socioeconomic gradient of child development: cross-sectional evidence from children 6-42 months in Bogota. J Human Resources 2015;50:464-83

46 Premand $\mathrm{P}$, Barry O. Behavioral change promotion, cash transfers and early childhood development: experimental evidence from a government program in a low-income setting. Washington D.C. 2020. Available: https://openknowledge.worldbank.org/handle/ $10986 / 34385$ 